\title{
SIZE OF THE BROAD LINE REGION IN NGC 3227: RESULTS FROM THE LAG CAMPAIGN *
}

\author{
I. SALAMANCA, T. BARIBAUD and D. ALLOIN \\ Observatoire de Meudon DAEC, 5 Place Jules Janssen, 92195 Meudon Cedex, France
}

We present the results of a monitoring campaign of the AGN in NGC 3227, conducted at La Palma by the LAG european consortium. The data are long slit spectra, obtained with the $1.5 \mathrm{~m}$ INT and $4.2 \mathrm{~m} \mathrm{WHT}$, from January to June 1990. The slit width was 1.5 arcsec. The spectral resolution was about $3 \mathrm{~A}$.

From the observed MgI $\lambda 5167,5173,5183$ in the NGC 3227 spectra we deduce that the stellar contribution is at most $40 \%$ at $\lambda 5180 \mathrm{~A}$ when the AGN is in his lower state.

To separate the BLR and NLR emissions we have performed a gaussian decomposition of the blends $\mathrm{H} \alpha+[\mathrm{NII}]+[\mathrm{SII}]$ and $\mathrm{H} \beta+$ [OIII]. We have constrained as many parameters as possible, following physical arguments. The same blend modeling was used for all epochs, only the amplitude of the BLR components was allowed to vary.

After having performed the line blend decomposition, subtracted the stellar population contribution and applied corrections for seeing differences, we have obtained the light curves for the BLR $\mathrm{H} \alpha$ and $\mathrm{H} \beta$ line emission as well as for the visible continuum (derived under the assumption that the NLR emission does not vary with time). We find out variation amplitudes around $40 \%$ over timescales of 6 weeks. The cross correlation of the line and continuum light curves provides a BLR size of $17+/-7$ days.

The Balmer line difference between the highest and lowest levels shows a symmetrical flat-topped profile with FWHM $\approx 2900 \mathrm{~km} / \mathrm{s}$. Assuming a keplerian broadening of the lines, and that the accretion disc is coplanar with the galactic disc, we conclude that the bulk of the BLR emission occurs at $10^{3}$ Schwarzschild radii and that the central object has a mass of $2 \times 10^{8} M_{o}$.

From a small sample of AGN (7) we find that the BLR size is proportional to $L^{\gamma}$, where $\gamma<0.5$, instead of 0.5 assumed in the models. A correlation analysis indicates a slope of about 0.2 .

\section{References}

Baribaud T., Salamanca I., Alloin D., Wagner S.,1993, 'Sources of uncertainty in the relative scaling of spectroscopic data', $A \& A S S$, in press.

Salamanca I., Alloin D., Baribaud T. et al., 1993, LAG paper, 'Spectroscopic monitoring of active galactic nuclei III. Size of the Broad Line Region in NGC 3227.', $A \& A$, in press.

* The Lovers of Active Galaxies (LAG) consortium was set up to apply for international time on the Canaries Observatories and conducted a series of programs related to the physics of AGN.

457

T. J.-L. Courvoisier and A. Blecha: Multi-Wavelength Continuum Emission of AGN, 457.

(c) 1994 IAU. Printed in the Netherlands. 